\title{
Optical property of Few-Mode Fiber with Non-uniform Refractive Index for Cylindrical Vector Beam Generation
}

\author{
Hongye Li ${ }^{\text {a }}$, Hongdan Wan* a, Zuxing Zhang* a, b, Bing Sun ${ }^{\text {a }}$, Lin Zhang, ${ }^{\text {a, }}$ \\ ${ }^{a}$ Nanjing University of Posts and Telecommunications, Nanjing 210003, China \\ ${ }^{\mathrm{b}}$ Aston Institute of Photonic Technologies, Aston University, Birmingham B4 7ET, UK
}

\begin{abstract}
This paper investigates optical properties of few-mode fiber with non-uniform refractive index, namely: the few mode fiber with U-shape refractive index and the two-mode and four-mode few-mode fiber with bent radius. Finite element method is used to analyze the mode distributions based on their non-uniform refractive index. Effective mode control can be achieved through these few mode fibers to achieve vector beam generation. Finally, reflection spectra of a few-mode fiber Bragg grating are calculated theoretically and then measured under different bending conditions. Experimental results are in good accordance with the theoretical ones. These few mode fibers show potential applications in generation of cylindrical vector beam both for optical lasing and sensing systems.
\end{abstract}

Keywords: few-mode fiber, cylindrical vector beam, non-uniform refractive index, few-mode fiber Bragg grating

\section{INTRODUCTION}

Cylindrical vector beams (CVBs) have drawn considerable attention recently for their symmetric intensity and polarization properties. The symmetrical polarization results in many applications, such as optical tweezers, surface plasmon resonance and material processing ${ }^{[1-3]}$. How to generate the CVBs including radically (TM01) and azimuthally (TE01) polarized beams has become a hot topic. Two methods are commonly used, namely the external-cavity ("passive") and the internal-cavity ("positive") method ${ }^{[4]}$. The former consist of spatial light modulator, wave plate and other spatial light units for generating spatial beams with symmetrical polarization ${ }^{[5,6]}$. The latter contains birefringence elements or few-mode fiber gratings (FMFG) as the mode selector to achieve high-order modes inside a fiber device, such as the few-mode fiber long period grating (FMF-LPG) and the few-mode fiber Bragg grating (FMF-BG) ${ }^{[7,8]}$

The FMF-LPG converts LP01 mode to LP11 modes by mechanical stress from special mechanical holders ${ }^{[7]}$, result in an increased dimension and higher probability of fiber damage. The FMF-BG is formed by a periodic index modulation over the photosensitive fiber core along propagation length, having the advantages of high-efficiency, low cost and all-fiber structure ${ }^{[8]}$. The forward propagating modes can be coupled to the phase-matched backward propagating modes, the fundamental mode inside the few-mode fiber is converted to the high-order modes, with modal properties determined by the index profile over the fiber core. However, UV-side-writing or fiber bending of actual used FMF brings birefringence and affects modal conversion due to the non-uniform refractive index profile over the fiber core. Since the high-order mode excitation and conversion efficiency decide the CVBs' generation efficiency, there still need efforts to investigate the optical property of a specially designed FMF with a non-uniform refractive index profile and to effectively achieve CVBs with higher mode purity.

In this paper, we investigate the optical properties of the FMFs with U-shape refractive index and bent FMF Bragg Grating (FMFBG). The reflection and transmission spectra of the FMFG are studied theoretically based on a 3dimensional non-uniform refractive index profile. Then the reflection spectra of the four-mode FMFBG and the transmission spectrum of the four-mode FMFBG as well as its modal profile are measured experimentally, high order modes could be excited and controlled. Finally, the reflection spectra under special bending condition are also given experimentally to achieve effective mode control.

*hdwan@njupt.edu.cn; *zxzhang@njupt.edu.cn; phone 86-13815898967; fax 86-25-85866296

Advanced Laser Manufacturing Technology, edited by Bingheng Lu, Huaming Wang, Proc. of SPIE Vol. 10153, 101530K · @ 2016 SPIE · CCC code: 0277-786X/16/\$18 · doi: 10.1117/12.2246037 


\section{MODAL PROPERTIES OF THE FMFS WITH NON-UNIFORM REFRACTIVE INDEX PROFILE}

\subsection{The U-shape refractive index FMF}

Figure 1 and Figure 2 illustrate the parameters of a U-shape refractive index FMF and its mode distribution profile at $1550 \mathrm{~nm}$, respectively. The LP11 mode is confined in the high refractive index ring and the fundamental mode power is extremely weak. Thus, the U-shape index FMF can be used to suppress the fundamental mode and excite the LP11 mode. With proper pumping method, this kind of fiber could be used for fiber lasers to generate CVBs.

\begin{tabular}{|c|c|c|c|c|}
\hline \multirow{6}{*}{0} & Name & Expression & Value & Description \\
\hline & r1 & 4[um] & $4 E-6 m$ & The radius of inner ring \\
\hline & r2 & 5.19 [um] & $5.19 \mathrm{E}-6 \mathrm{~m}$ & The radius of outer ring \\
\hline & r3 & 62.5 [um] & $6.25 \mathrm{E}-5 \mathrm{~m}$ & The radius of cladding \\
\hline & n1 & 1.45 & 1.45 & The refractive index of cladding \\
\hline & n2 & 1.4682 & 1.4682 & The refractive index of ring \\
\hline
\end{tabular}

Fig1.Parameters and refractive index profile of the U-shape refractive index FMF

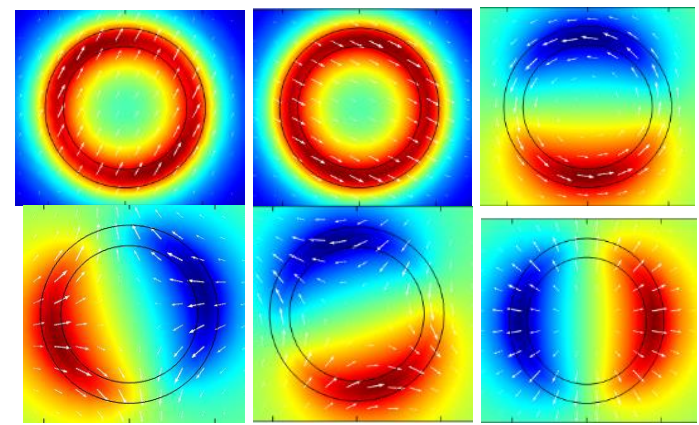

Fig.2 Mode distribution profile of U-shape refractive index FMF at 1550nm

\subsection{The bent FMF}

The refractive index of bent fibers can be calculated using the Marcuse formula: $n^{\prime}=n(1+x / R)$, where $n$ is refractive index of core or cladding, $R$ is the bending radius[4]. Table.1 demonstrates the modal profile of a two-mode fiber (with normalized frequency $\mathrm{V}=6.4$ ) with different bending radius. Obviously, higher modes dissipate prior to lower modes when the curvature radius continues to be decreased. Therefore, small bending results in suppression of the high-order modes first, with the fundamental mode or low-order modes retain, which is testified experimentally in the following part of this paper. Table 1 and 2 show the calculated modal profile of the two and four mode fibers with different bending radius. It can be seen that, a reduced bending radius results in decreased modes' number being confined inside the bent fiber core.

Table.1 Modal profile of the two-mode FMF with different bending radius. Re: Reserve; BD: Begin Dissipating; D: Dissipate

\begin{tabular}{|c|c|c|c|c|}
\hline Curvature Radius R/cm & LP01 & LP11 & & \\
& & & & \\
\hline 10 & $\mathrm{Re}$ & $\mathrm{Re}$ & $\mathrm{Re}$ & $\mathrm{BD}$ \\
\hline 5 & $\mathrm{Re}$ & $\mathrm{Re}$ & $\mathrm{BD}$ & $\mathrm{D}$ \\
\hline 4 & $\mathrm{Re}$ & $\mathrm{Re}$ & $\mathrm{D}$ & $\mathrm{D}$ \\
\hline 2 & $\mathrm{Re}$ & $\mathrm{D}$ & $\mathrm{D}$ & $\mathrm{D}$ \\
\hline 1 & $\mathrm{D}$ & $\mathrm{D}$ & $\mathrm{D}$ & $\mathrm{D}$ \\
\hline
\end{tabular}


Table. 2 Modal profile of the four-mode FMF with different bending radius. When the curvature radius is $4 \mathrm{~cm}$, only the four lowest modes (LP01, LP11, LP21, LP02) exist. Re: Reserve; BD: Begin Dissipating; D: Dissipate

\begin{tabular}{|c|c|c|c|c|c|c|}
\hline $\begin{array}{c}\text { Curvature radius } \\
\mathrm{R} / \mathrm{cm}\end{array}$ & $\mathrm{LP01}$ & & $\mathrm{LP11}$ & & & \\
& & & & & & \\
\hline 10 & $\mathrm{Re}$ & $\mathrm{Re}$ & $\mathrm{Re}$ & $\mathrm{Re}$ & $\mathrm{Re}$ & $\mathrm{BD}$ \\
\hline 4 & $\mathrm{Re}$ & $\mathrm{Re}$ & $\mathrm{Re}$ & $\mathrm{Re}$ & $\mathrm{D}$ & $\mathrm{D}$ \\
\hline 3 & $\mathrm{Re}$ & $\mathrm{Re}$ & $\mathrm{Re}$ & $\mathrm{D}$ & $\mathrm{D}$ & $\mathrm{D}$ \\
\hline 2 & $\mathrm{Re}$ & $\mathrm{Re}$ & $\mathrm{D}$ & $\mathrm{D}$ & $\mathrm{D}$ & $\mathrm{D}$ \\
\hline 1 & $\mathrm{D}$ & $\mathrm{D}$ & $\mathrm{D}$ & $\mathrm{D}$ & $\mathrm{D}$ & $\mathrm{D}$ \\
\hline
\end{tabular}

\section{OPTICAL PROPERTIES OF THE FMFBG}

\subsection{Operation principle}

Modal coupling process decides the reflection spectra of a specific FMFBG. The coupling intensity is decided by the overlap integral $K_{k j}^{t}(z)=\frac{\omega}{4} \iint_{c o r e} \Delta \varepsilon(x, y, z) e_{k t}(x, y) e_{j t}(x, y) d x d y$, where $e_{k t}(x, y)$ and $e_{j t}(x, y)$ are the normalized electric field of mode $\mathrm{k}$ and mode $\mathrm{j}$, respectively. The resonance wavelength is decided by the phase matching formula:

$\beta_{k}-\beta_{j}=\frac{2 \pi}{\Lambda}$, where $\beta_{k}, \beta_{j}$ and $\Lambda$ are the propagation constant of mode $\mathrm{k}$, propagation constant of mode $\mathrm{j}$ and period of grating [5]. The coupling process occurs when the forward mode and backward mode satisfy phase matching formula in fiber Bragg grating. For self-coupling, the phase matching formula can be simplified as $\lambda_{B}=2 n_{\text {eff }} \Lambda$ ( $\lambda_{B}$ and $n_{\text {eff }}$ are the resonance wavelength and effective refractive index, respectively), the simplified formula is $\lambda_{B}=\left(n_{\text {eff }, 1}+n_{\text {eff }, 2}\right) \Lambda$.

\subsection{Reflection spectra of the two-mode FMFBG}

The grating period of the two-mode FMFBG is 533nm. Fig. 3(a) and (b) illustrates the fiber dispersion curve and reflection spectra under different mode-ratio (the mode intensity ratio between LP01 mode and LP11 mode). Three reflection peaks represent the self-coupling and mutual-coupling between the LP01 and LP11modes.The reflection peak intensity is decided by the mode-ratio, increased intensity of single mode results in enhanced self-coupling. 


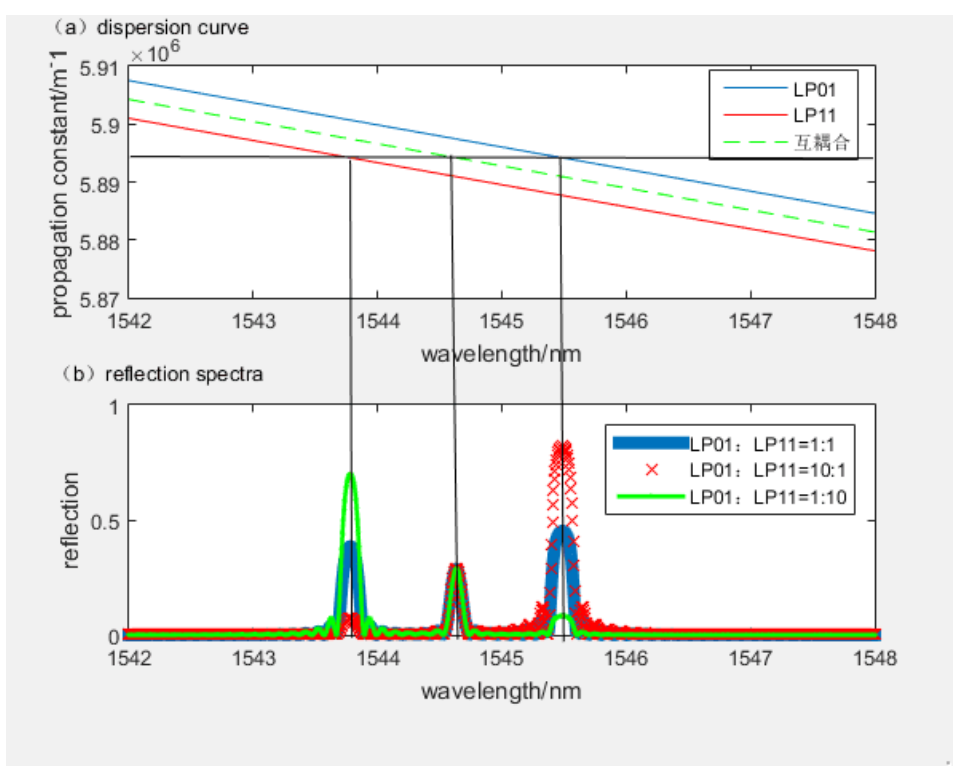

Fig.3 Optical dispersion curves and reflection spectra of the two-mode FMFBG with different mode-ratio: Blue, LP01:LP11=1:1; Red, LP01:LP11=10:1; Green, LP01:LP11=1:10

\subsubsection{Reflection spectra of the four-mode FMFBG}

The calculated dispersion curve and reflection spectra of the four-mode FMFBG (with normalized frequency V=6.08 and grating period $=533 \mathrm{~nm}$ ) are presented in Fig.4. There are 10 peaks in the reflection spectra (4 self-coupling peaks and 6 mutual-coupling peaks).

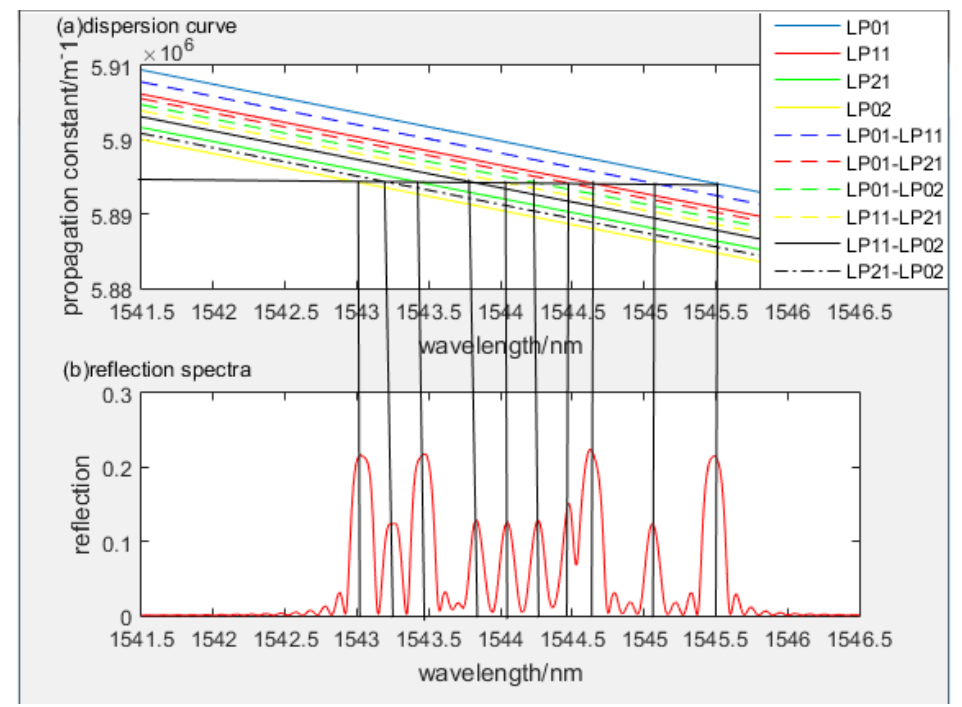

Fig.4 Optical dispersion curves and reflection spectra of the four-mode FMFBG

\subsection{Experimental results and discussion}

Figure 5 illustrates the measured reflection spectra of our self-made three different FMFBGs: a step-index four-mode FMFBG, a graded-index four-mode FMFBG and a step-index two-mode FMFBG, respectively. 

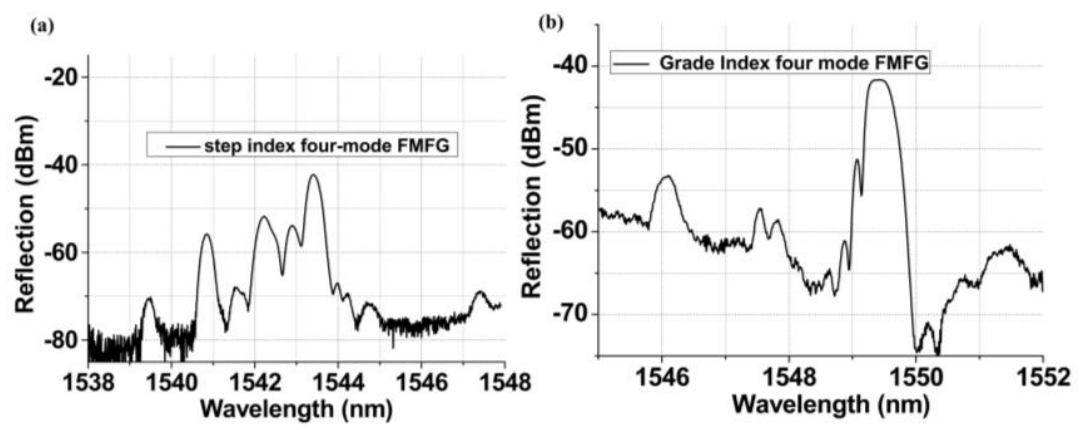

Fig.5 Reflection spectra of: (a) the step-index four-mode FMFBG; (b) the graded-index four-mode FMFBG with $2.5 \mu \mathrm{m}$ offset splicing to the SMF

Figure 6 shows the measured transmission spectra of the step-index two-mode FMFBG and the modal profile (measured by a NIR-CCD) near three transmission-dip wavelengths. According to the theoretical analysis, three kinds of mode-coupling process are involved, namely the self-coupling of the LP01 mode (1551.54 nm); the mutual-coupling between the LP11 and LP01 modes (1550. $90 \mathrm{~nm})$ and the self-coupling of the LP11 mode (1549.79 nm). In the inset picture (iii), the LP01 mode is reflected and the LP11 mode transmits through the FBG and is detected by the CCD However, because of the non-uniform refractive index profile over the fiber core, modal profile of the LP11 mode takes on a two-lobe shape, rather than a doughnut-shape, which is in good accordance with the simulations. CVB can be achieved using this two-mode FMFBG near the self-coupling wavelength of the LP01 mode.

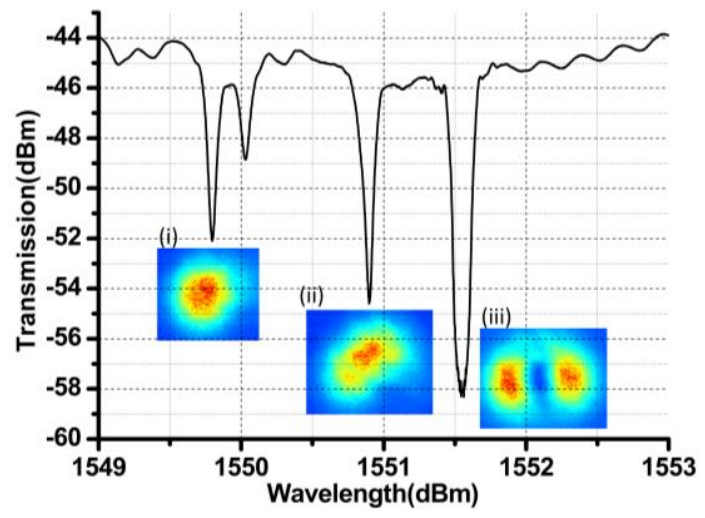

Fig. 6 The measured transmission spectrum of the step-index two-mode FMFBG, the inset picture shows the modal profile near three different transmission-dip wavelengths

Figure 7 shows different reflection spectra of the bent step index four-mode FBG with curvature radius increased from $R_{l}$ to $R_{6}$. High order modes are excited through off-set splicing method as shown in top of Fig.7 and several peaks in the FMFBG's reflection spectra appear. The number of these resonant peaks is reduced with the bent radius reduced. When the curvature radius is $\mathrm{R}_{1}$, only fundamental mode can be excited, with more than one resonant peak appears in the reflection spectra. Due to the non-uniform cross refractive index profile, energy can be transferred from one mode to the other modes. These agree well with our theoretical analysis: higher modes lose energy but lower modes retain within bent FMFs, which offers an effective method to excite and control high order mode for generation of the CVBs. 


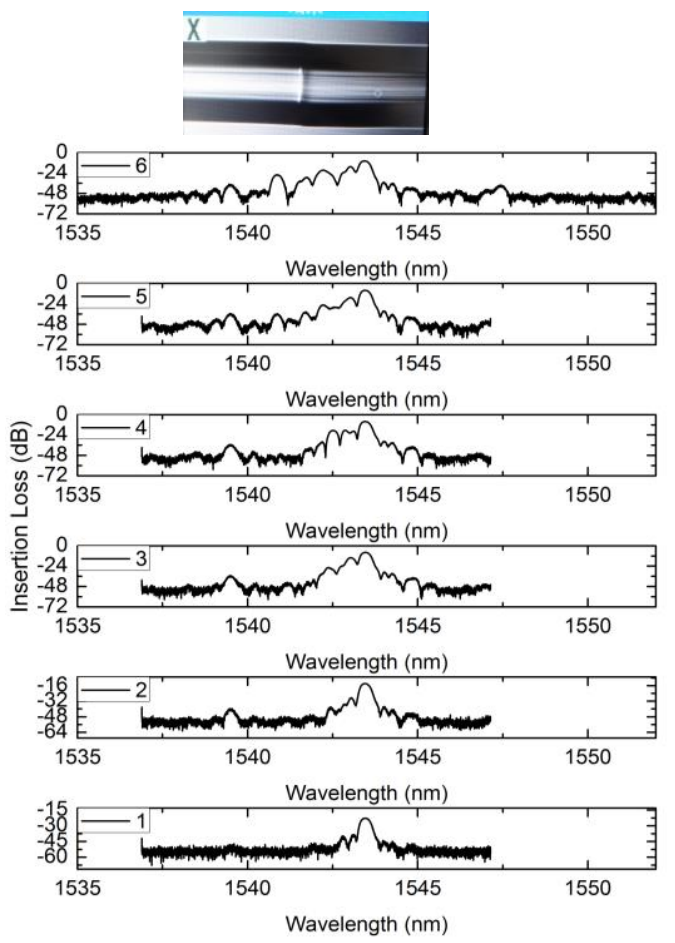

Fig.7 Reflection spectra of the bent four-mode FMFBG with different curvature radius. High order mode is excited through an $3 \mu \mathrm{m}$ offset-splicing method.

\section{CONCLUSION}

In conclusion, we investigate the optical properties of FMF with non-uniform refractive index profile theoretically and experimentally. For the U-shape refactive index FMF, if applied in fiber laser, CVBs are generated by adjusting pump power; For the bent FMFs, high optical modes in the FMF can be effectively suppressed through decreasing curvature radius; For the FMFBGs, the number of reflection peaks decreases with minimized curvature radius. Based on the FMFs with specially designed refractive index profile, we can control the mode distribution of FMFs effectively and realize required CVBs in an all-fiber, flexible manner. These few-mode fibers show potential applications in generating cylindrical vector beam both for optical lasing and sensing systems.

\section{ACKNOWLEDGMENT}

This work is supported by National Science Foundation of Jiangsu Province (No. BK20150858), P. R. China and NUPTSF (Grant No. NY214059 and NY213083).

\section{References}

[1] Y. Kozawa, S. Sato, etc "Optical trapping of micrometer-sized dielectric particles by cylindrical vector beams" Opt. Express, 18(10):10828-10833 (2010).

[2] W. Chen, Q. Zhan, etc "Realization of evanescent Bessel beam via surface plasmon interference excited by a radially polarized beam" Opt. Letters, 34(6):722-724 (2009).

[3] M. Meier, etc "Material processing with pulsed radially and azimuthally polarized laser radiation" Applied physics amaterials Science \& Processing, 86:329-334 (2007).

[4] Y. Kozawa, S. Sato, etc "Generation of a radially polarized laser beams by use of a conical Brewster prism", Opt. Letters 30(22):3063-3065 (2005).

[5] Y. Li, J. G. Hu, etc, "Characteristic analysis of two-mode fiber Bragg grating", App. Physics, 117(4),1221-1228 (2014). 
[6] M. M. Ali, Y. Jung, K. Lim, etc "Characterization of mode coupling in few-mode fiber Bragg gratings with selective mode excitation". IEEE Photon.Tech. Letters, 27(16):1713-1716(2015).

[7] Y. H. Zhao, Y. Q. Liu, L. Zhang, etc, "Mode converter based on the long-period fiber gratings written in the twomode fiber", Opt. Express 24(6): 6186- 6195 (2016)

[8] B. Sun, A. T. Wang, etc "Low-threshold single-wavelength all-fiber laser generating cylindrical vector beams using a few-mode fiber Bragg grating", Opt. Letters, 37(4), 464-466(2012) 